Noname manuscript No.

(will be inserted by the editor)

\title{
Consistency of seven different GNSS global ionospheric mapping techniques during one solar cycle
}

\author{
David Roma-Dollase • Manuel \\ Hernández-Pajares · Andrzej \\ Krankowski - Kacper Kotulak • Reza \\ Ghoddousi-Fard · Yunbin Yuan · Zishen \\ Li · Hongping Zhang · Chuang Shi · \\ Cheng Wang • Joachim Feltens · \\ Panagiotis Vergados - Attila Komjathy • \\ Stefan Schaer - Alberto García-Rigo • \\ José M. Gómez-Cama
}

Received: date / Accepted: date

\author{
Abstract In the context of the International GNSS Service (IGS), several \\ IGS Ionosphere Associated Analysis Centers (IAAC) have developed different \\ techniques to provide Global Ionospheric Maps (GIMs) of Vertical Total Elec- \\ Manuel Hernández-Pajares, Alberto García-Rigo \\ Universitat Politècnica de Catalunya (UPC-IonSAT), Barcelona, Spain \\ E-mail: manuel.hernandez@upc.edu \\ David Roma-Dollase, José M. Gómez-Cama \\ Department of Engineering: Electronics, Universitat de Barcelona, Barcelona, Spain \\ Andrzej Krankowski, Kacper Kotulak \\ Space Radio-Diagnostics Research Centre (SRRC/UWM), University of Warmia and \\ Mazury in Olsztyn, Olsztyn, Poland \\ Reza Ghoddousi-Fard \\ Canadian Geodetic Survey, Natural Resources Canada, Canada \\ Yunbin Yuan \\ Institude of Geology and Geophysics, Chinese Academy of Sciences, China \\ Zishen $\mathrm{Li}$ \\ Academy of Opto-Electronics, Chinese Academy of Sciences, China \\ Hongping Zhang, Chuang Shi, Cheng Wang \\ Wuhan University, China \\ Joachim Feltens \\ Telespazio-VEGA Deutschland GmbH, c/o European Space Operations Centre, European \\ Space Agency, Germany \\ Attila Komjathy, Panagiotis Vergados \\ Jet Propulsion Laboratory, National Aeronautics and Space Administration, United States \\ Stefan Schaer \\ Center for Orbit Determination in Europe, Switzerland
}


tron Content (VTEC) since 1998. In this paper we present a comparison of the performances of all the GIMs created in the frame of IGS. Indeed we compare the classical ones (for the ionospheric analysis centers CODE, ESA/ESOC, JPL and UPC) with the new ones (NRCAN, CAS, UWH). To assess the quality of them in fair and completely independent ways, two assessment methods are used: a direct comparison to altimeter data (VTEC-altimeter) and to the difference of slant total electron content (STEC) observed in independent ground reference stations (dSTEC-GPS). The main conclusion of this study, performed during one solar cycle, is the consistency of the results between so many different GIM techniques and implementations.

Keywords Global Navigation Satellite Systems · Ionosphere · Global Ionospheric Maps · Vertical Total Electron Content · Model Validation

\section{Introduction}

Global Navigation Satellite Systems (GNSS) have opened a large set of new applications during the last two decades. One of them, based on the frequencydependent code delay and phase advance when traveling trough the Earth ionosphere, is its continuous global sounding. In particular, the computation and assessment of VTEC GIMs from the dual-frequency measurements, have deserved an important effort. Indeed, estimation techniques have been developed based, among others, on VTEC expansion in terms of spherical harmonics or electron content described in few layers or voxels in some cases. Assessments based on independent measurements of VTEC and STEC variation have provided a fair ranking and optimal weights for combination (see introduction and references in [22]). Moreover additional recent works are focused on GIM assessment from vessels, [27], at mid and low latitude GNSS receivers, [1], and looking the apparent stability of associated Differential Code Biases but not assuring an independent assessment, [32].

Two independent and external techniques to assess in a fair way the global VTEC ionospheric models computed from GNSS data, are applied in this paper in the context of the IGS [4]:

1. VTEC reference values from dual-frequency altimeter measurements over the oceans.

2. STEC difference values from GIM-independent dual-frequency GPS measurements.

The assessed GIMs were computed, on the one hand, by CODE, ESA, JPL and UPC. These are analysis centers contributing since 1998.5 and their GIMs are used by the University of Warmia-Mazury, UWM, since 2007, to generate the IGS combined GIM. On the other hand NRCan has resumed the GIM delivery to IGS during beginning of 2016, at the same time that Chinese Academy of Sciences (CAS) and Wuhan University (WHU) have become new contributors. To distinguish the GIMs corresponding to different centers, a four digits identification is used (more details will be found in section 3.1), which can include as well information about some non standard general characteristics: 
- The second digit indicates a different GIM rate than the default one of two hours: Q for "quarter of an hour rate, and "H" for hour rate.

- The third digit indicates a "rapid" IGS latency (one day) when it is equal to "R".

- The fourth digit indicates the GIM scope (typically "G" from "global").

After the introduction, the manuscript is organized in four additional sections devoted to a short overview on the different ionospheric modeling techniques to be compared, the assessments versus VTEC-altimeter and dSTECGPS independent measurements and finally the conclusions and recommendations.

\section{GNSS global ionospheric modelling techniques under comparison}

The GIMs produced by the following seven IAAC have been assessed in this work:

- Center for Orbit Determination in Europe (CODE) with the CODG GIM.

- European Space Agency/European Space Operations Centre (ESA/ESOC) with the ESAG GIM.

- Jet Propulsion Laboratory (JPL) with the JPLG GIM.

- Universitat Politècnica de Catalunya (UPC-IonSAT) with the UPCG and the recent UQRG GIMs.

- Chinese Academy of Sciences (CAS) with the CASG GIM.

- Natural Resources Canada (NRCan) with the EMRG GIM.

- Wuhan University with the WHRG and WHUG GIMs.

All the previous GIMs assessed are computed under the final (longest production) latency, except for EMRG, UQRG and WHRG (formerly known as WHUB), which are rapid products with latencies below two days. Moreover the final combined IGS GIM (IGSG, see [19]) has been evaluated as well.

More details can be found in next subsections, summarized in Table 1.

\subsection{Center for Orbit Determination in Europe}

The Center for Orbit Determination in Europe (CODE) has been contributing to the IGS ionosphere analysis activities since the establishment of the IGS ionosphere working group in 1998 (see [34], [33]). At that time, the IONosphere Map Exchange (IONEX) Format was developed under the lead of members of CODE in order to get an IGS-approved data format for comparison and combination of GPS-derived ionosphere maps [35]. Inclusion of GLONASS data for operational ionosphere analysis (and POD) could be accomplished in 2003. VTEC maps are generated on a daily basis using GPS/GLONASS data from about 300 globally distributed stations of the IGS and other institutions. The VTEC is modeled in a solar-geomagnetic reference frame using a spherical harmonics expansion up to degree and order 15. Piece-wise linear functions 
are used for representation in the time domain. The time spacing of their vertices is 1 hour, corresponding to the epochs of the VTEC maps. A modified single-layer model mapping function approximating the JPL three-shell model is used. Instrumental biases with respect to each involved GNSS code observable are estimated as constant values for each day. They are modeled with one set of bias parameters for each satellite and each station in case of GPS and for each satellite-station link in case of GLONASS [3]. Discontinuities at day boundaries are minimized by performing an extra analysis step (at normal-equation level): the provided CODE GIM (CODG) results correspond to the results for the middle day of a 3-day combination analysis solving for 73 times 256, or 18688 VTEC parameters and 3 daily sets of GNSS code bias parameters. CODE GIM products are available from three analysis lines: final, rapid, and predicted (GIM predictions are computed based on rapid and final GIM results). All these products are provided in IONEX format. In addition, content-reduced versions are generated by estimating $(4+4)$ Klobuchar-style ionospheric coefficients best fitting the CODE IONEX maps of each analysis line.

\subsection{European Space Agency/European Space Operations Centre}

ESA/ESOC contributes with IONEX products to the IGS Ionosphere Working Group since its inception in 1998. Global Ionospheric TEC \& RMS Maps (GIMs) are routinely delivered to the IGS in final mode in 2-hour time resolution with 11 days latency and in rapid mode in 2-hour and 1-hour time resolution with 1 day latency. In August 2013 ESOCs ionosphere processing software IONMON (IONosphere MONitoring facility) became an integral part of ESOCs NAPEOS (NAvigation Package for Earth Orbiting Satellites,http://www. esa.int/Our_Activities/Operations/N/ Since then, GLONASS data are processed in combination with GPS data. It is planned to include in future also Galileo and further other Global Navigation Satellite System (GNSS) data into the IONMON processing.

Currently (October 2017), ESA's GIMs (ESAG) are still computed with a single layer approach (IONMON Version 1) [5], taking slant range TEC observables derived from dual-frequency GPS and GLONASS data, whereby ionospheric TEC is modelled by spherical harmonics in combination with a daily Differential Code Biases (DCBs) fitting. In this single layer approach, the ionospheric TEC is assumed to be condensed on a hollow sphere enveloping the Earth at an altitude of typically $450 \mathrm{~km}$. On that sphere, the global TEC distribution is then described by a degree and order 15 spherical harmonics function. The function coefficients are determined from GPS and GLONASS dual-frequency data recorded at about 300 globally distributed ground sites in final mode processing and from 200250 sites in rapid mode processing. GIM fitting is conducted, depending on final or rapid mode settings, in a 2-hour or 1-hour time resolution. Normal equations of the previous fits are included into the current hourly/2-hourly update with weights decaying exponentially with time to stabilize the actual fit; all normal equations of one day are combined 
with equal weights to fit a daily set of DCBs for all satellites and all stations. In order to avoid singularity for this DCBs fit, the DCB reference is set to zeromean over all satellites. The Modified Single Layer Model Mapping Function (MSLM) [33] is employed to map slant range TEC observables, derived from the GPS and GLONASS data, into the vertical. This is the same mapping function that is also used by CODE. Model-internal, all computations are conducted in solar-geomagnetic reference system.

There are no investigations to develop or enhance the single layer model further. Instead, actual activities focus on the establishment of a new 3D modelling approach (IONMON Version 2) having further than only GNSS-derived TEC observables processing capability, e.g. ionosonde, radio occultation data, enhanced time resolutions and real-time capacity, which shall replace the classical single-layer model. The new 3D model development is actually in the coding and testing stage. In addition, this new 3D approach will be augmented by a new model for the plasmasphere/plasmapause currently developed in a cooperation project with Deutsches Zentrum fr Luft- und Raumfahrt (DLR) Neustrelitz (more details can be found in [8] and [9]). The current single layer model processing and software will only retained as long as the new 3D model is not yet operational.

\subsection{Jet Propulsion Laboratory}

The Jet Propulsion Laboratory (JPL) has been providing global ionospheric maps ([30]) to IGS since 1998, in the commonly adopted IONEX format, for operational ionosphere, altimetry, and Precise Orbit Determination (POD) applications. From 1998 until 2015, JPL was only using GPS observables to produce the global TEC maps. Subsequently, the inclusion of GLONASS observables was completed in early 2015. Similarly to the rest of the IGS centers, JPL generates VTEC maps on a daily basis using GPS/GLONASS data from $200+$ globally distributed stations of IGS and other institutions. The JPL/GIM software uses the so-called zeta function to select the ground-based stations that provide maximum global coverage based on their spatial distribution. The JPL-produced IONEX maps are provided with a 15-minute temporal resolution and a varying spatial resolution (typically at $5^{\circ} \times 5^{\circ}$, or better). Initially, the JPL/GIM software approximated the ionosphere using the widely accepted single-shell model. However, in early 2000s [25] introduced a modified model that approximates the ionosphere using a three-shell model, with each shell centered at a fixed altitude $(250,450$, and $800 \mathrm{~km}$, respectively) with a constant electron number density. GIM is a data-driven model that uses a Kalman filter-based approach to fit the observed slant TEC (STEC) observations from the ground-based receivers to a model that is described by a set of horizontal basis function coefficients (e.g. [28];[29], [25], [24]). A single bias for each GPS satellite and each receiver is estimated when using GPS observables alone. When using GLONASS observables, JPL/GIM estimates a separate bias for each GLONASS receiver-to-satellite pair because 
each GLONASS satellite transmits at a different frequency channel, and thus there is generally a distinct receiver bias for each GLONASS satellite pair. All instrumental biases are considered constants during the day and are estimated together with the basis coefficients as nuisance parameters. To ensure smoothness of the modeled VTEC arcs at the day boundaries, the JPL/GIM products use additional observations of six-hour lengths prior and after the day of the estimated VTEC parameters, solving in total of $36(=6+24+6)$ times 256, or 9,216 VTEC parameters. A detailed description of the method of using the GPS and GLONASS measurements to produce the GIM global ionospheric TEC maps, we refer the reader to [36].

\subsection{Universitat Politècnica de Catalunya}

The Tomographic Ionosphere model software (TOMION) has been developed at UPC since 1995 under the initiative of the second author of this manuscript ([15],[16]). The first versions were focused on over-passing some fundamental limitations of the classical estimation of the electron content from dualfrequency GPS measurements.

Indeed, the unrealistic assumption of the electron content distribution in a single-layer at a constant effective height, distorting the VTEC estimates, is fixed in TOMION by using a multilayer voxel model. In particular the assumption of two layers of voxels, to describe the electron density, remove this effect when ground-based data is only used (Figure 2 in [16]). The tomographic model is then solved with a Kalman filter.

Moreover, the dependence of the classical electron content estimation on pseudorange measurements (affected extensively by multipath) and its dependence on the associated interfrequency instrumental delays, significantly affect the STEC calibration as well (see Figure 3 in [23]). This second source of error is avoided by using only the geometry-free combination of carrier phase measurements, whose ambiguities are properly estimated in the Kalman filter, separately from the mean electron density, thanks to the change of geometry along the continuous phase arcs.

Since then, TOMION has been evolving in different versions able to process both ground based GNSS ionospheric data, GPS LEO radio occultation data ([20], [17]), and ionospheric corrections for precise user positioning (Wide Area Real-Time Kinematic [18]).

UPC GIMs, generated with TOMION, started in June 1st, 1998. They contribute since the beginning of the IGS Ionospheric working group combined product, in the role of UPC as one of the IAAC ([19]). The interpolation of the individual VTEC estimates, computed from the tomographically callibrated STECs, was improved from a two hour resolution splines-based interpolated GIM (UPCG) to a fifteen-minutes resolution kriging-based interpolation (UQRG, [31]). 


\subsection{Chinese Academy of Sciences}

The approached used by CAS for generating the GIM product is SHPTS which means Spherical Harmonic function Plus generalized Trigonometric Series [26]. Based on SHPTS, the calculation is carried out by the following two steps, firstly, the variation of ionospheric TEC in global and local scales are individually model by the SH function with the order and degree of 15 and the generalized TS function over each station based on the thin-layer approximation. The generalized TS function was developed by [38] based on the triangular series function proposed by [7]. It has been demonstrated that GTS can effectively describe the subtle variation that occurs in ionospheric TEC using data obtained over a single-day period. The generalized TS function consists two parts shown by next equation.

$$
V=\sum_{n=0}^{n_{\max }} \sum_{m=0}^{m_{\max }}\left\{E_{n m}\left(\phi-\phi_{0}\right)^{n} h^{m}\right\}+\sum_{k=0}^{k_{\max }}\left\{C_{k} \cos (k \cdot h)+S_{k} \sin (k \cdot h)\right\}
$$

Where $V(\phi, h)$ denotes the vertical ionospheric TEC; $\phi$ and $h$ denote the geographic latitude of ionospheric IPP (intersecting pierce point) and current local time of IPP; $\phi_{0}$ denotes the geographic latitude of station; $n_{\max }$ and $m_{\max }$ denotes the maximum degree of polynomial development; $k_{\max }$ denotes the maximum degree of finite Fourier series; $E_{n m}, C_{k}$ and $S_{k}$ denotes the coefficients of local ionospheric model to be estimated. In the local ionospheric modeling, a simplified mapping function $M(z)$ is applied to convert the line-ofsight ionospheric TEC (STEC) to vertical direction (VTEC) at the ionospheric IPP, as shown by next equation.

$$
\left\{\begin{array}{c}
S=M(z) \cdot V \\
M(z)=\left[1-\frac{\sin ^{2} z}{\left(1+H_{\mathrm{ion}} / R_{E}\right)^{2}}\right]^{-\frac{1}{2}}
\end{array}\right.
$$

Where $z$ is the satellites zenith angle at station; $H_{\text {ion }}$ is the altitude of ionospheric thin layer; $R_{E}$ is the mean radius of the Earth.

Secondly, the vertical ionospheric TEC and its RMS at each grid point in GIM product is calculated by combing the global and local ionospheric TEC models by the improved DADS (different areas different stations) method ([37]) shown by next two equation.

$$
\begin{gathered}
V_{i}=\left\{\begin{array}{cc}
V_{g, i} & M=0 \\
\frac{\sum_{m=1}^{M} P_{m} \cdot V_{s, i, m}}{\sum_{m=1} P_{m}} & M>0
\end{array}\right. \\
\sigma_{i}=\left\{\begin{array}{cc}
\sigma_{g, i} & M=0 \\
\sqrt{\frac{\sum_{m=1}^{M} P_{m} \cdot \sigma_{s, i, m}^{2}}{\sum_{m=1}^{M} P_{m}}} & M>0
\end{array}\right.
\end{gathered}
$$

Where $V_{i}$ and $\sigma_{i}$ are the SHPTS-based ionospheric vertical TEC and its RMS at the i-th grid point respectively; $M$ is the number of available stations 
which is near the i-th grid point defining as that the stations to which the elevation of the corresponding grid point is greater than $25^{\circ} ; V_{g, i}$ and $\sigma_{g, i}$ are the ionospheric VTEC and its RMS at the i-th grid point calculated from the SH-based global ionospheric TEC model; $V_{s, i, m}$ and $\sigma_{s, i, m}$ are the ionospheric TEC and RMS at the i-th grid point calculated from the GTS-based local ionospheric TEC model obtained at the m-th station; $P_{m}$ is the weight of $V_{s, i, m}$ and $\sigma_{s, i, m}$, as it is shown in next equation.

$$
P_{m}=\frac{1}{\sigma_{0, m}^{2} \cdot\left[\cos ^{2}(\epsilon)+1\right]}
$$

Where $\epsilon$ is the elevation of grid point corresponding to the location of $\mathrm{m}$-th station; $\sigma_{0, m}$ is the variance of unit weight for the local ionospheric model and can be obtained in the least square estimation. It can be seen from previous equations that the ionospheric vertical TEC at the grid point near from the GNSS stations is estimated from the corresponding local ionospheric models. If more than one local ionospheric model is selected for this calculation, the ionospheric TEC would be estimated by adjusting those from each local ionospheric model using an elevation-dependent weight. Generally, the local ionospheric model is more accurate than the global ionospheric model, thus only those local ionospheric TEC models are used to improve the accuracy of ionospheric TEC estimates around the contributed stations. In addition, there may be some discontinuities between some neighbor grid points (for instance one with $\mathrm{M}=0$ and the other with $\mathrm{M}_{\dot{\iota} 0} 0$ ), but this situation only occurs over the border of the lands and oceans, and basically not affected the overall performance of GIM.

\subsection{Natural Resources Canada Global Ionospheric Model}

Canadian Geodetic Survey of Natural Resources Canada (NRCan) has resumed the submission of VTEC GIMs to IGS data centers since April 2015. NRCan GIMs used to be contributing to the IGS combined GIMs since its inception until 2003. NRCan daily global maps, named with its former name as EMRG, are generated using GNSS phase smoothed geometry-free pseudo range measurements (including GLONASS since November 2016) on a single layer model from around 350 stations. Satellite differential code biases are estimated at each station on a daily basis with a zero mean condition. VTEC at IPPs are gridded in a geomagnetic reference frame and are represented using spherical harmonic coefficients of degree and order 15 in 1 hour intervals. [10] Apart from global daily maps presented in this paper, NRCan has also developed a number of other products from GNSS sensing of the ionosphere including:

- Regional near real-time and daily VTEC maps represented using spherical cap harmonic analysis that covers Canada and adjacent regions. [11]

- Near real-time global VTEC maps (15 minute intervals) from GNSS realtime IGS (RT-IGS) stations. 
- Monitoring ionospheric irregularities using high rate $(1 \mathrm{~Hz})$ GNSS phase rate measurements of RT-IGS stations. [12]

\subsection{Wuhan University}

The University of Wuhan is using an expansion in terms of Spherical Harmonics, similarly to CODE and ESA to represent global ionospheric VTEC maps. Wuhan University as a new member of IAACs use these functions for GIM computation as well. VTEC modeling with ground-based GNSS dualfrequency observations has been one of hot topics in GNSS. However, most GNSS stations are located on the mainland, only a small part of them are scattered on some islands in the oceans. As a result, the accuracy of ionospheric mapping over these areas will be very poor and negative VTEC values may appear too. To solve this problem, [28] used climatological ionospheric models to simulate data to mitigate significant gaps between measurements. [31] adopted kriging interpolation technique to update VTEC maps, which has been shown to be a good approach. Wuhan University proposed directly implementing the mathematically equivalent of non-negative constraints to reconstruct VTEC models [39]. In other words, Wuhan reformulated the VTEC mapping problem as an inequality-constrained Gauss-Markov model. In order to make sure that the estimated results are physically meaningful, such prior constraints should be directly incorporated as part of the measurement system. Wuhan solves a least square problem with inequality constraints, which is also called inequality-constrained least squares adjustment in Geodesy. The results have shown that the new algorithm efficiently eliminates the unwanted behavior of negative VTEC values. In future, Wuhan will continue to focus on providing better and faster global ionospheric products by using multi-GNSS measurements.

\section{VTEC-altimeter assessment}

The direct VTEC measurements, obtained by dual-frequency altimeters like TOPEX, JASON and JASON2, among others ( $V_{a}$ in TECUs), provide a GNSS-independent reference data very useful to assess the performance of GIMs in some of the most challenging scenarios: over the oceans, typically far from the permanent GNSS receivers contributing to the GIM estimation (see for example [19], and in more detail [22]). It is computed from the vertical phase ionospheric delay provided in Ku-band frequency $\left(I_{\mathrm{Ku}}\right.$, for $f_{\mathrm{Ku}}=13.575 \mathrm{GHz}$ ). This measurement is obtained from the dual-frequency altimeter observations over the oceans, as follows:

1. The reflections on the ice, marked in the input data, are discarded.

2. The isolated set points, showing jumps regarding consecutive observed VTEC values bigger than 20 TECUs, are also rejected. 
Table 1 Summary of the different GIMs assessed in this work.

\begin{tabular}{|c|c|c|c|c|c|}
\hline $\begin{array}{l}\text { GIM } \\
\text { ID }\end{array}$ & Method & $\begin{array}{c}\text { DCB } \\
\text { Computation } \\
\end{array}$ & $\begin{array}{l}\text { Shell } \\
\text { Model }\end{array}$ & $\begin{array}{l}\text { Start } \\
\text { Date }\end{array}$ & Ref. \\
\hline IGSG & Weighted Mean & Combined & $\begin{array}{l}\text { Com- } \\
\text { bined }\end{array}$ & 1998.4 & [19] \\
\hline CODG & Spherical Harmonics & $\begin{array}{l}\text { Same time as } \\
\text { VTEC }\end{array}$ & $\begin{array}{l}\text { Modified } \\
2-\mathrm{D}\end{array}$ & 1998.4 & [33] \\
\hline $\mathrm{ESAG}^{1}$ & Spherical Harmonics & $\begin{array}{l}\text { Same time as } \\
\text { VTEC }\end{array}$ & $2-\mathrm{D}$ & 1998.4 & {$[5]$} \\
\hline JPLG & Three-shell Model & $\begin{array}{l}\text { Same time as } \\
\text { VTEC }\end{array}$ & $3-\mathrm{D}$ & 1998.4 & {$[28]$} \\
\hline UPCG & Tomographic with splines & From VTEC & $3-\mathrm{D}$ & 1998.4 & {$[16]$} \\
\hline $\mathrm{UQRG}^{2,3}$ & Tomographic with kriging & From VTEC & $3-\mathrm{D}$ & 2011 & {$[31]$} \\
\hline CASG & $\begin{array}{l}\text { Spherical Harmonics and Gener- } \\
\text { alized Trigonometric Series }\end{array}$ & $\begin{array}{l}\text { Same time as } \\
\text { VTEC }\end{array}$ & $2-\mathrm{D}$ & 2016 & {$[26]$} \\
\hline $\mathrm{EMRG}^{3}$ & Spherical Harmonics & $\begin{array}{l}\text { Same time as } \\
\text { VTEC }\end{array}$ & $2-\mathrm{D}$ & $\begin{array}{l}1998.4, \\
2015.3\end{array}$ & [10] \\
\hline $\mathrm{WHRG}^{3}$ & $\begin{array}{lrr}\text { Spherical Harmonics } & \text { and } \\
\text { Inequality-constrained } & \text { Least } \\
\text { Squares } & & \\
\end{array}$ & $\begin{array}{l}\text { Same time as } \\
\text { VTEC }\end{array}$ & $2-\mathrm{D}$ & 2016 & {$[39]$} \\
\hline WHUG & $\begin{array}{lrr}\text { Spherical Harmonics } & \text { and } \\
\text { Inequality-constrained } & \text { Least } \\
\text { Squares } & & \\
\end{array}$ & $\begin{array}{l}\text { Same time as } \\
\text { VTEC }\end{array}$ & $2-\mathrm{D}$ & 2016 & {$[39]$} \\
\hline
\end{tabular}

1 A 3D multi-layer assimilation model is currently under development by ESA. 2 The time interval of UQRG is fifteen minutes, in contrast with the other GIMs which have a time interval of two hours. 3 UQRG, EMRG and WHRG are rapid GIMs with a latency of less than two days, in contrast with the other final GIMs which have a latency about one week.

3. Afterwards we smooth the VTEC measurements with a sliding window of $n=16$ consecutive samples, see Eqs. 6 and 7 .

4. In case of a lack of consecutive observations, for time spans larger than 9 seconds, the smoothing is reinitialized.

$$
V_{a}=\frac{1}{n} \sum_{i=1}^{n} v_{a}\left(t_{i}\right)
$$

where

$$
v_{a}=-\frac{f_{\mathrm{Ku}}^{2}}{40.3 \cdot 10^{16}} I_{\mathrm{Ku}}
$$

In order to assess the performance of the GIMs with the VTEC-altimeter data we have analyzed one whole solar cycle, from day 180 of year 2001 to day 007 of year 2016. This means more than 190 million dual-frequency TOPEX, JASON-1 and JASON-2 ionospheric delay observations were processed and compared with the corresponding values provided by the different GIMs in IONEX format applying the most accurate temporal (in local-time) and spatial (second order) interpolation algorithm among the ones suggested in [35].

Indeed, Figure 1 shows the mean value of the VTEC measurements throughout each day, along all the analyzed period. This corresponds to different 


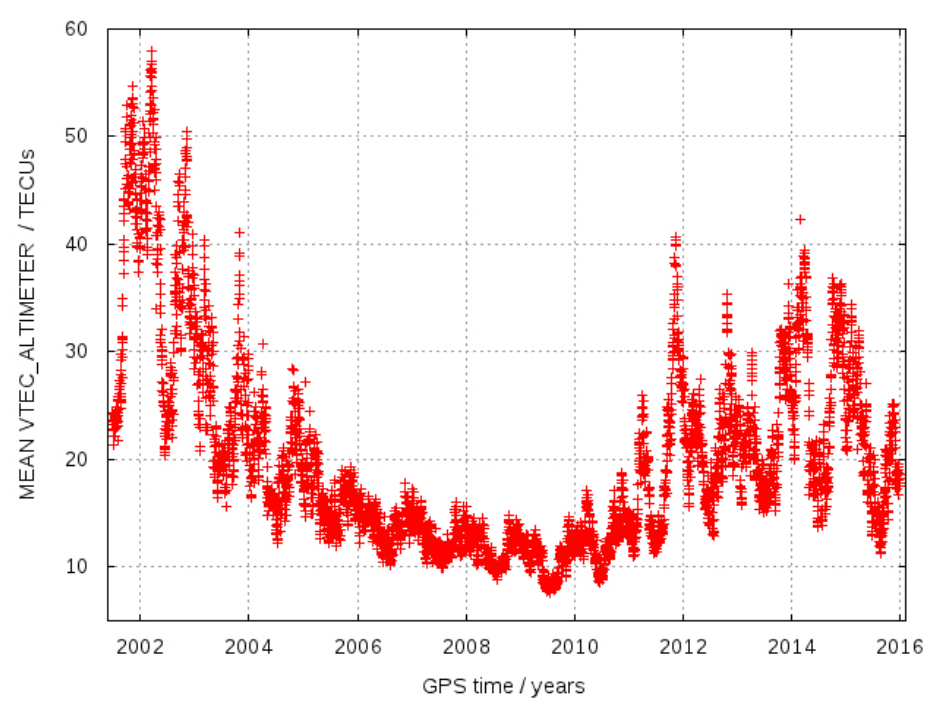

Fig. 1 Daily altimetric mean VTEC during a whole solar cycle: Days 180, 2001 to 007, 2016 (+190 million dual-frequency TOPEX, JASON-1 and JASON-2 ionospheric delay observations processed)

altimeters: TOPEX, JASON-1 or JASON-2, using the newest one when available. The solar cycle and seasonal VTEC periodic modes can be clearly seen, in agreement with previous works (see for instance [19]).

Since the altimeter on-board the satellites will not include the total electron content present inside the upper region of the ionosphere and plasmasphere, there is a small bias, in the order of a few TECUs [14], between the measurements of the VTEC of the GIMs and the dual-frequency altimeters. In this sense, to compare the performance of the GIMs the standard deviation of the difference between the VTEC of the GIMs and the VTEC obtained from the dual-frequency altimeters is preferred and will be discussed below, since it will minimize this difference [22].

Furthermore, in Figure 2 we can see the remarkable agreement of the bias, at one to few TECUs level, regarding the altimeter VTEC for most of the IAACs. This happens among the different mapping functions used, related with the general leveling, and despite the well known small calibration error of the altimeters (excess of few TECUs, [2]). Moreover the "climatology" of the topside electron content between the altimeter and GNSS orbits, in terms of seasonal and solar cycle periods, can be clearly seen, in agreement with previous studies such as [13].

The discrepancies of all available IGS VTEC GIMs vs +190 millions of altimeter direct VTEC measurements over the seas during the last 15 years, have been analyzed. In Figure 3, two sets of GIMs have been compared: 

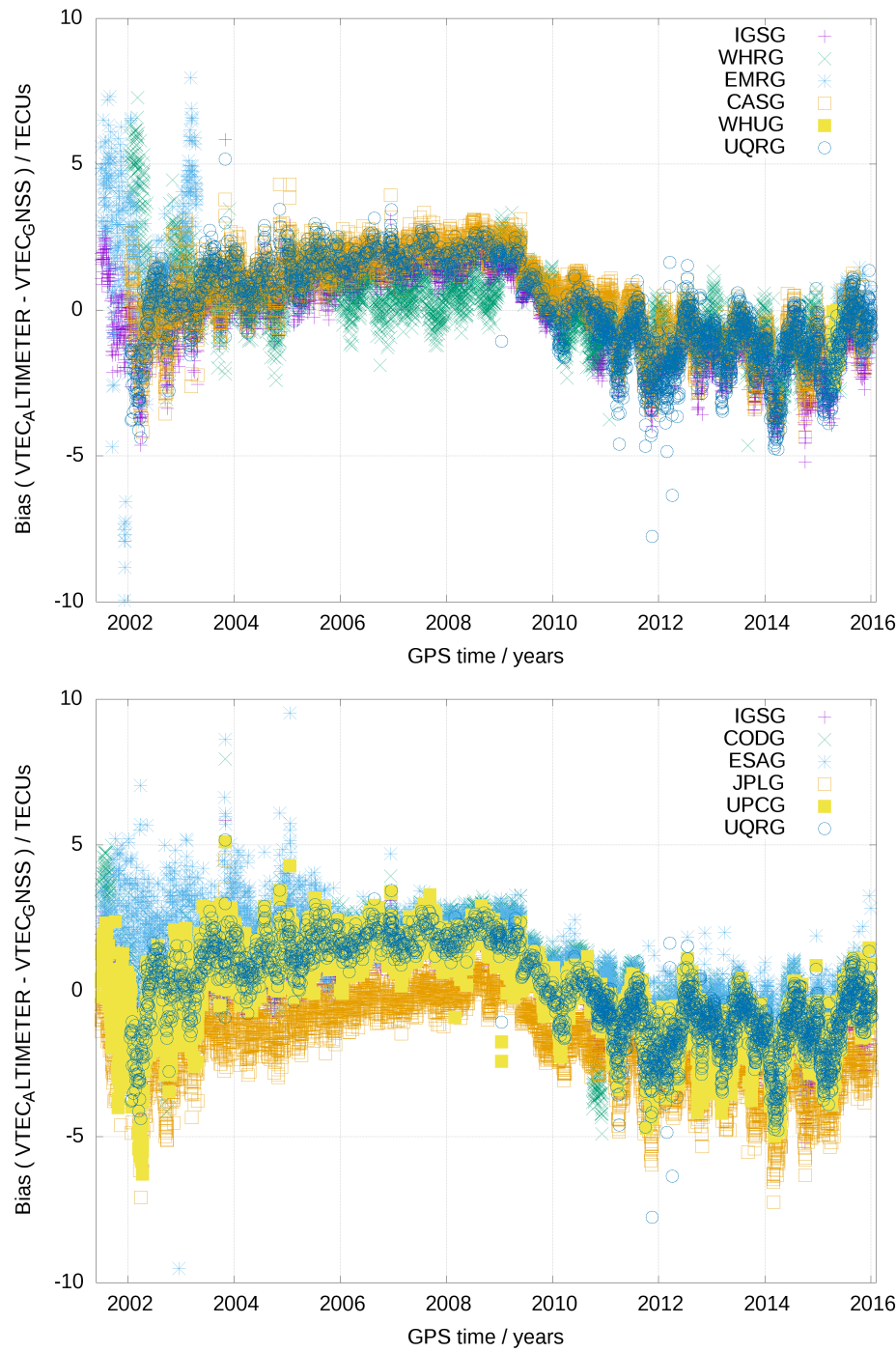

Fig. 2 Daily mean (or bias) of the altimeter-VTEC minus the corresponding GIM-VTEC, involving new GIMs (top plot) and classical GIMs (bottom plot). The comparison has been performed over the same dataset referenced in Figure 1.

- Set\#1 with the IAAC contributing to IGS since 1998.4 (CODG, ESAG, JPLG and UPCG), the recent GIM from UPC (UQRG) and the IGS combined GIM (IGSG).

- Set\#2 with the new IAAC contributing to IGS (CASG,WHRG and WHUG) or resuming their contribution (EMRG), UQRG and IGSG. 

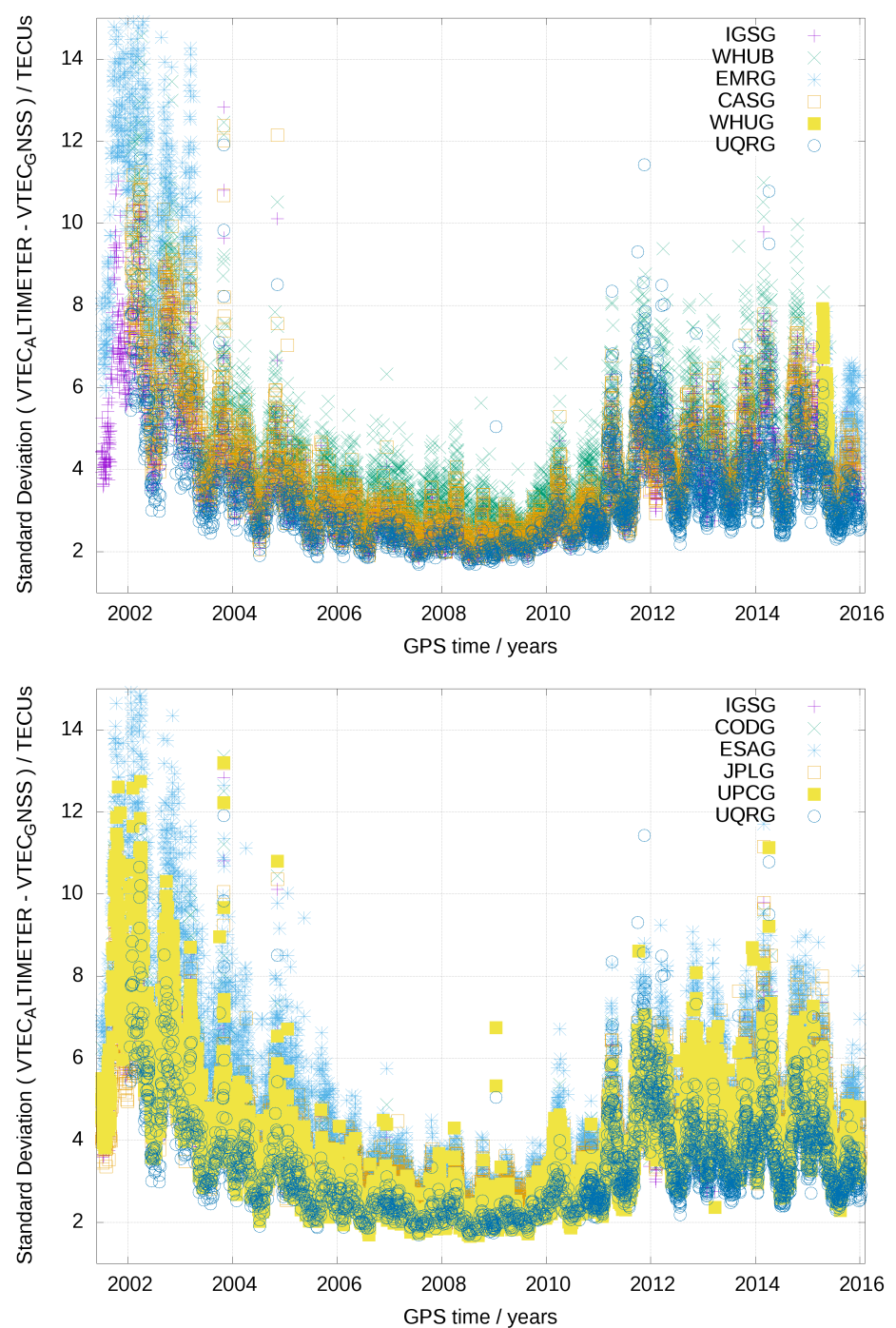

Fig. 3 Daily standard deviation of the VTEC difference regarding altimeter VTEC measurements, involving new GIMs (top plot) and classical GIMs (bottom plot). The comparison has been performed over the same dataset referenced in Figure 1.

An overall general agreement is found between the techniques of the seven analysis centers, with VTEC daily standard deviations typically ranging from three to ten TECUs, depending on the solar cycle phase.

These results are confirmed in detail in the histograms, Figure 4, corresponding to the previous time series of standard deviation of the GIM VTEC versus altimeter VTEC for the whole solar cycle analyzed. The results are summarized in Table 2. Still, for some GIMs, like WHUG and EMRG, there is 
Table 2 Summary of the GIMs assessment vs +190 millions of altimeter VTEC measurements, including an overall period of up to almost 5000 days and a common period of 21 days (remarks: [*] For the newest period of EMRG product submission to IGS only, days 117-365, 2015; [**] Very limited sample).

Up to more than 1 solar cycle, within 21 common days, within

days 180,2001 to 007,2016 117,2015 to 007,2016

\begin{tabular}{|c||ccc||cc|}
\hline $\begin{array}{c}\text { GIM } \\
\text { Id. }\end{array}$ & \# days & $\begin{array}{c}\text { Std. Dev. } \\
\text { / TECU }\end{array}$ & $\begin{array}{c}\text { Rel. Error } \\
\%\end{array}$ & $\begin{array}{c}\text { Std. Dev. } \\
\text { / TECU }\end{array}$ & $\begin{array}{c}\text { Rel. Error } \\
\%\end{array}$ \\
\hline IGSG & 4927 & 3.9 & 19.9 & 4.6 & 21.1 \\
CODG & 4934 & 4.3 & 22.0 & 4.8 & 21.8 \\
ESAG & 4926 & 5.3 & 26.6 & 5.6 & 25.5 \\
JPLG & 4912 & 4.1 & 21.2 & 4.8 & 21.9 \\
UPCG & 4925 & 3.9 & 19.7 & 4.2 & 19.1 \\
CASG & 4914 & 3.9 & 20.9 & 4.6 & 21.1 \\
EMRG & $255[*]$ & $(4.8)$ & $(26.2)$ & 5.9 & 26.5 \\
WHRG & 4416 & 4.6 & 24.8 & 5.5 & 25.0 \\
WHUG & $42[* *]$ & $(5.9)$ & $(26.9)$ & 5.5 & 25.0 \\
UQRG & 3063 & 3.6 & 17.8 & 3.6 & 16.3 \\
\hline
\end{tabular}

a more limited amount of data. This is the reason why there is only a common set of 21 days for all the GIMs, which are explicitely compared in the second part of the table. As we can see both time spans show a similar trend. This is specially relevant for WHUG where the amount of data is statistically speaking very low an it is more difficult to extract any trend. For EMRG on the other hand the situation is not so critical and it can be assessed that the results are clearly in the range of results of the classical IGS GIMs. Furthermore, an interesting factor to assess the quality of the GIMs from the histogram is the number of counts of the tail of the histogram. For most GIMs we can see a fast decay of the counts from the histogram, leaving the number of counts below the $10 \%$ of the peak value of the standard deviation between 6 TECUs and 8 TECUs. The only exception of this, without taking into account the GIMs with a more limited number of samples, is ESAG which only reaches this 10 $\%$ of the peak value between 10 TECUs and 12 TECUs.

A summary of the GIMs assessment vs altimeter VTEC can be found in Table 2. Relative errors from $26 \%$ to $18 \%$ have been found among the different GIMs for more than 13 years. One question which arises in this point is the impact on the accuracy of the time interval to which the GIM is provided. This is answered in the next sub-section.

\subsection{Influence of GIM update time rate}

In order to assess the influence of the GIM time sampling in the VTEC error, we have compared the one- and two-hour GIMs from UPC: UHRG and U2RG respectively. These are obtained downsampling the UQRG one, originally com- 

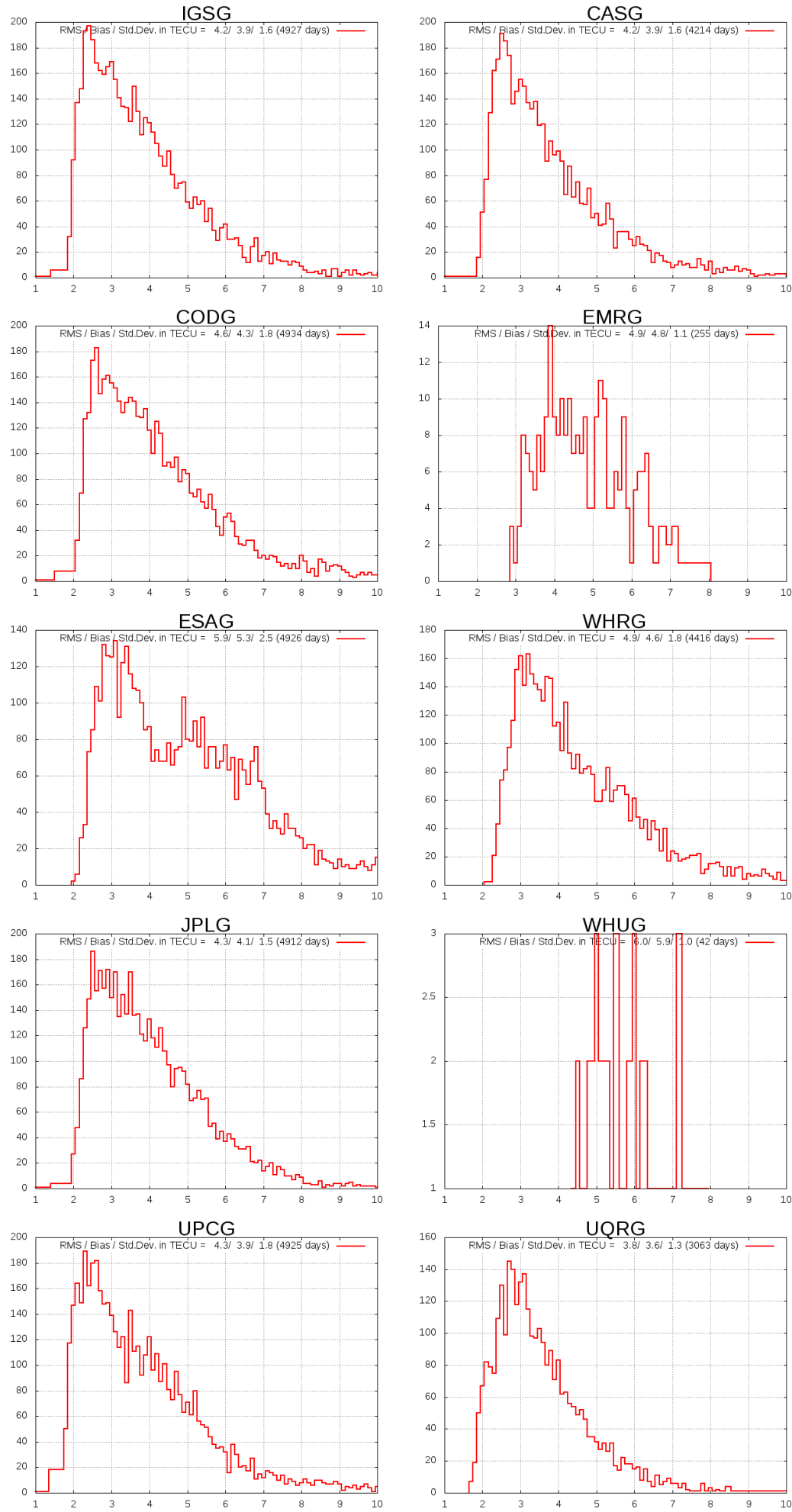

Fig. 4 Histogram (counts, in $\mathrm{Y}$-axis) representing the distribution of the standard deviation of the altimeter-VTEC minus the corresponding GIM-VTEC (horizontal axis in TECUs), involving classical GIMs (left column) and new GIMs (right column). The comparison has been performed with the available GIMs during a whole solar cycle: Days 180, 2001 to 007, 2016 (+190 million dual-frequency TOPEX, JASON-1 and JASON-2 ionospheric delay observations processed) 

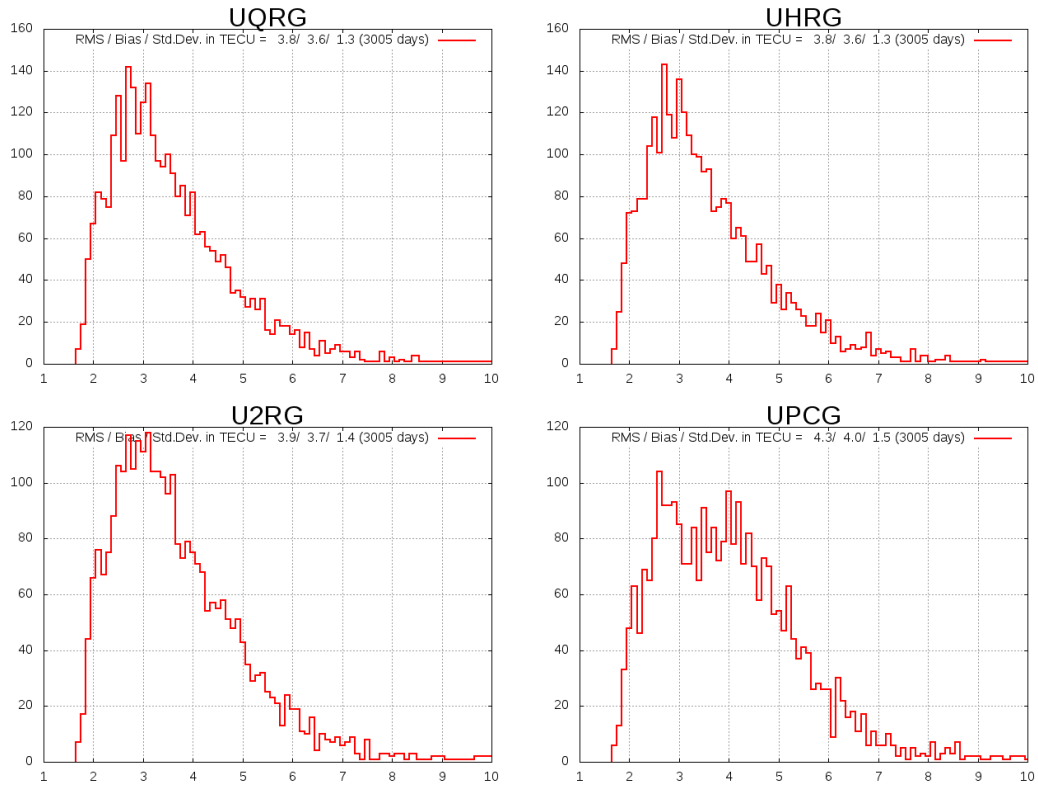

Fig. 5 Histogram (counts, in Y-axis) of the daily standard deviation VTEC residual of different UPC GIMs regarding to altimeter VTEC external values, TOPEX, JASON-1 and JASON-2 (horizontal axis in TECUs) for a common set of 3005 days since 2002 to 2015 (see more details in the text).

Table 3 Summary of the influence of the GIM sampling time and technique change exemplified in four different UPC GIMs (see details in the text): overall standard deviation of VTEC residuals regarding to altimeter VTEC external values (TOPEX, JASON-1 and JASON-2) for a common set of 3005 days since 2002 to 2015.

\begin{tabular}{|cc|}
\hline $\begin{array}{c}\text { GIM } \\
\text { Id. }\end{array}$ & $\begin{array}{c}\text { Std. Dev. } \\
\text { / TECU }\end{array}$ \\
\hline UQRG & 3.6 \\
UHRG & 3.6 \\
U2RG & 3.7 \\
UPCG & 4.0 \\
\hline
\end{tabular}

puted each 15 minutes. UQRG combines tomography with splines-based and kriging interpolation, with the official final UPC GIM, UPCG not using kriging interpolation. It is shown (Figure 5 and Table 3 ) that the GIM time interval between 15 min to 2 hours has little influence, when the recommended quadratic interpolation in latitude and local-time ([35], third page) is performed.

However the change of technique can have a noticeable influence, as it is shown just under a change in the interpolation scheme as it is shown in Table 3 , from splines (UPCG) to kriging (U2RG), both at two hours time interval.

The results of the relative error vs the altimeter-GPS VTEC are similar to the one given in Table 3 . 


\section{4 dSTEC-GPS assessment}

The GPS ionospheric carrier phase difference, $\Delta L_{I}$, corrected from carrier phase windup for a given pair transmitter-receiver, provides a very precise ionospheric reference. Indeed, this is the case when we consider the difference regarding the value corresponding to the highest elevation $\left(E_{\max }\right)$ line-of-sight and along a phase-continuous arc of data (i.e. without cycle-slips in between). The resulting observed magnitude is the corresponding STEC variation in space and time (dSTEC, $\Delta S$ in next equation):

$$
\Delta S=S(t)-S\left(t_{\text {Emax }}\right)=\frac{1}{\alpha}\left(L_{I}(t)-L_{I}\left(t_{E_{\max }}\right)\right)
$$

where $\alpha=\frac{q^{2}}{3 \pi^{2} m_{\mathrm{e}} \epsilon_{0}} \simeq 40.3$ in S.I. units, being $q$ and $m_{\mathrm{e}}$ the charge and mass of the electron, respectively, and $\epsilon_{0}$ the dielectric constant in the vacuum (see more details in [22]).

Such directly observed dSTEC value is typically more accurate than 0.1 TECU (the measurement -thermal noise and multipath- error) and independent of any assumption or model (see more details in [6]). In this sense, dSTEC provides an excellent electron content reference in the (slant) transmitterreceiver line-of-sight, very useful to assess and compare the performance of different ionospheric models. The benefit of the dSTEC assessment is that it covers the corresponding VTEC, the mapping function and their time evolution ([21]). Therefore, it complements the VTEC-altimeter assessment previously presented.

In order to perform the dSTEC-GPS assessment on the same GIMs previously assessed vs VTEC-altimeter, a set of 60 worldwide GNSS receivers, not used by any of the different GIMs to evaluate, have been selected (see Figure 6). Such receivers have been chosen guaranteeing the most feasible homogeneous distribution during two solstice and two equinox days in 2015: 082, 146,280 and 330.

A first overall summary of the results is shown in Figure 7, where the dSTEC relative error vs receiver latitude is represented for the classical IGS GIMs (top plot) and the new IGS GIMs (bottom plot).

A clear influence in terms of latitude is shown for different GIMs. Among the expected degradation at low latitude, in coincidence with the equatorial anomaly peaks, some GIMs degrades significantly at the Southern Hemisphere. This effect can be clearly seen in the spatial distribution of dSTEC relative error shown in next maps of Figure 8.

The summary of these results are given in Table 4 in terms of the overall relative error, expressed as a percentage of the ratio between the GIM dSTEC error root mean square (RMS) and the observed dSTEC RMS, and the number of receivers multiplied by the number of days processed. The external dSTECGPS assessment is qualitatively quite compatible with the one provided by VTEC-altimeters (Table 3) but with two main differences: 


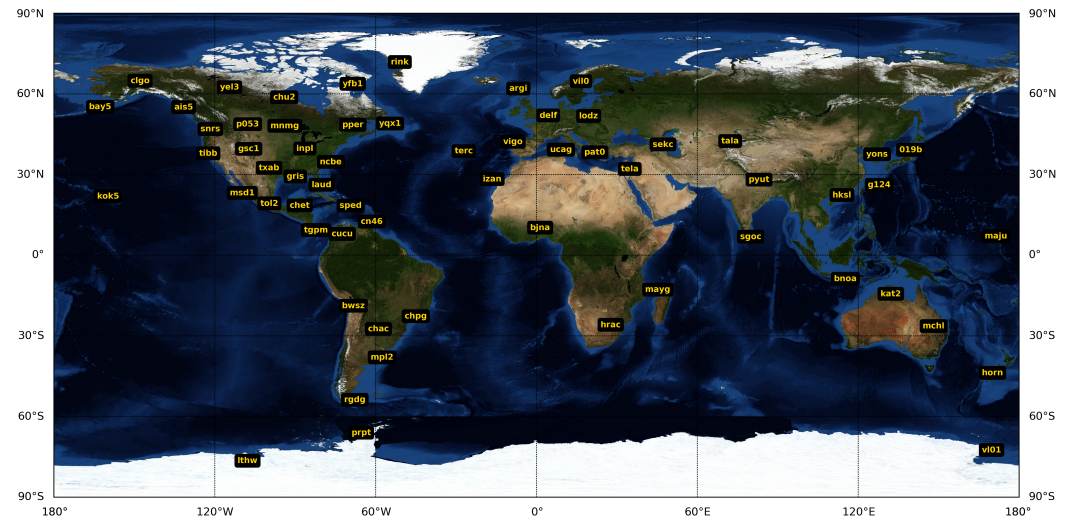

Fig. 6 Map of independent GNSS receivers selected to perform the dSTEC-GPS assessment during two solstice and two equinox days in 2015: 082, 146, 280 and 330.
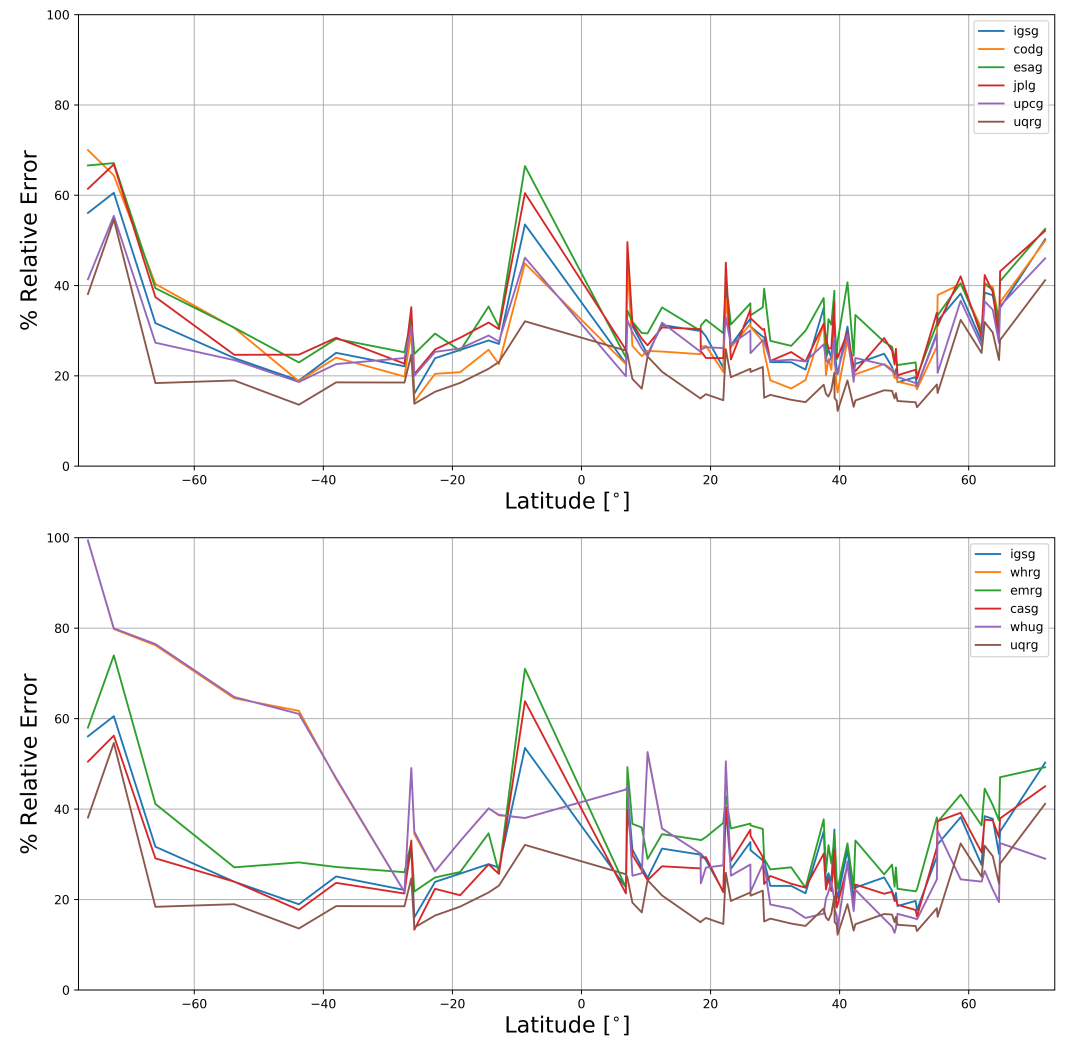

Fig. 7 dSTEC relative error vs latitude for classical IGS GIMs (top plot) and new ones (bottom plot), for +50 independent GNSS receivers during two solstice and two equinox days in 2015: $082,146,280$ and 330 


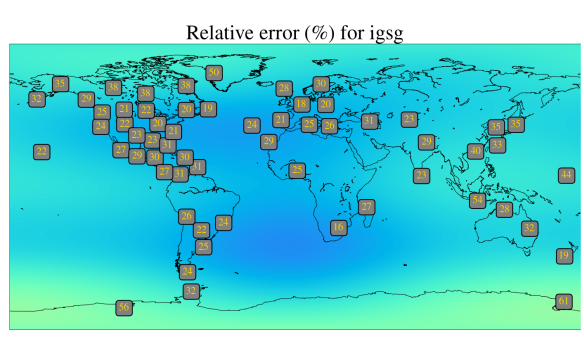

Relative error (\%) for codg

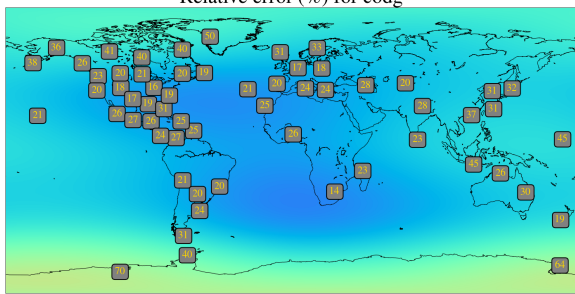

Relative error (\%) for esag

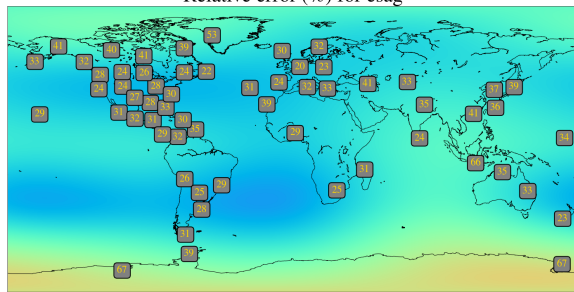

Relative error (\%) for jplg

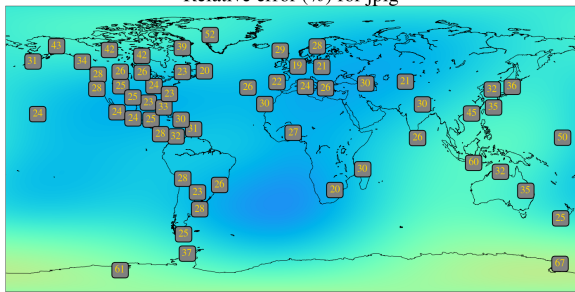

Relative error (\%) for upcg

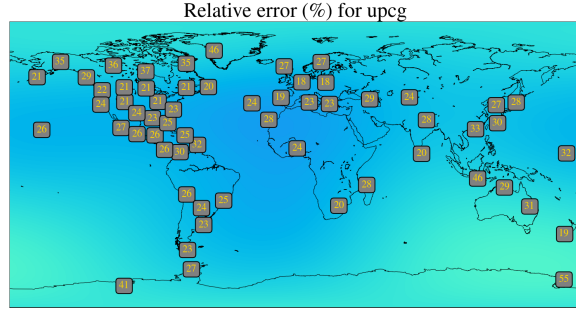

Relative error (\%) for casg

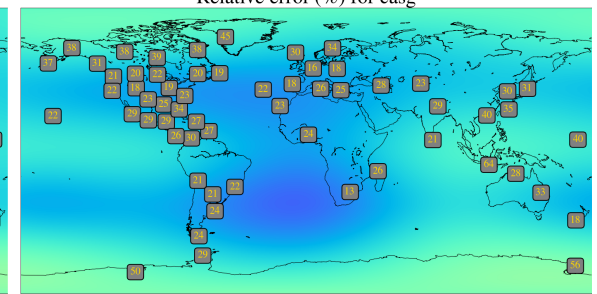

Relative error (\%) for emrg

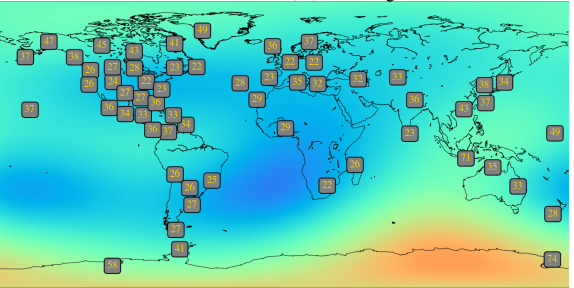

Relative error (\%) for uqrg

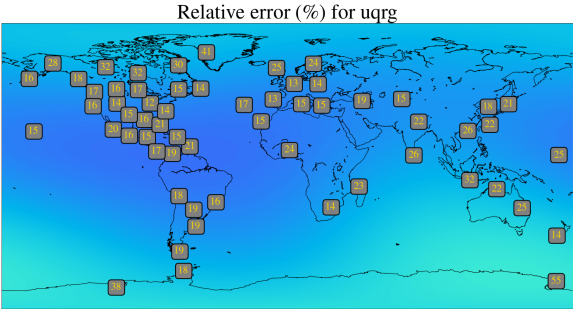

Relative error (\%) for whub

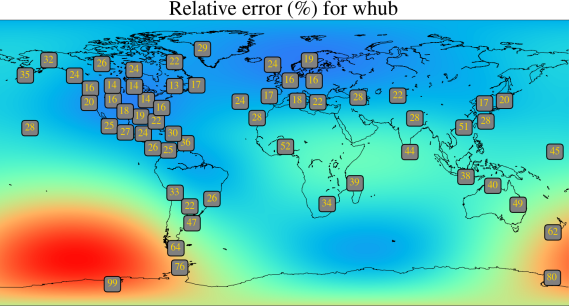

Relative error (\%) for whug

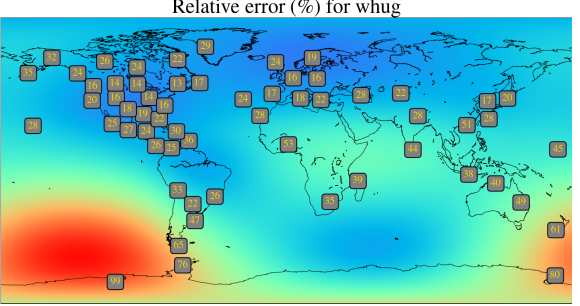

Fig. 8 Map approximating the spatial distribution of dSTEC relative error for classical IGS GIMs (left hand column, for IGSG, CODG, ESAG, JPLG and UPCG from top to bottom) and new GIMs (right hand one, for CASG, EMRG, UQRG, WHRG and WHUG from top to bottom), for +50 independent GNSS receivers during two solstice and two equinox days in 2015: 082, 146, 280 and 330. Color red represents an error of $100 \%$ and dark blue of $0 \%$. 
Table 4 Summary of the dSTEC relative error for classical and new IGS GIMs, including the number of receiver*days available and processed in each case, within the +50 independent GNSS receivers for days 082, 146, 280 and 330 of 2015, with Kp indices in the ranges $2.3-4.3,0.3-2.3,3.7-7.3$ and $0.3-1.3$, respectively (remarks: [*] number of available independent GNSS receivers significantly smaller than those of IGSG, CODG, ESAG, UPCG and UQRG; [**] extremely limited number of independent GNSS receivers available for dSTEC assessment compared with the other GIMs).

\begin{tabular}{|ccc|}
\hline $\begin{array}{c}\text { GIM } \\
\text { Id. }\end{array}$ & $\begin{array}{c}\text { Rel. Error } \\
\text { dSTEC / } \%\end{array}$ & \# Rec. ${ }^{*}$ Days \\
\hline IGSG & 28.9 & 238 \\
CODG & 27.8 & 238 \\
ESAG & 33.0 & 238 \\
JPLG & $(31.0)$ & $180[*]$ \\
UPCG & 26.9 & 238 \\
CASG & $(28.0)$ & $178\left[^{*}\right]$ \\
EMRG & $(33.6)$ & $178\left[^{*}\right]$ \\
WHRG & $((30.7))$ & $60\left[^{* *}\right]$ \\
WHUG & $((30.7))$ & $60[* *]$ \\
UQRG & 20.5 & 233 \\
\hline
\end{tabular}

1. The relative errors appear larger; this can be related with small reference values of dSTEC, when the given observation is not far from the highestelevation (i.e. the reference one).

2. For some centers the dSTEC-GPS results changes regarding to the VTECaltimeter one. This might be due to a less realistic mapping-function used in the GIM estimation (dSTEC-GPS is very sensitive to this), or to a most reduced statistics. This can affect JPLG, CASG and EMRG, but especially WHRG and WHUG (see again Table 3) which dSTEC performance numbers should not be considered directly comparable with the ones of the remaining analysis centers.

\section{Conclusions}

Global VTEC Ionospheric maps provided by seven IAACs have been analyzed in two different ways and scenarios, always versus external reference data, providing very consistent results between them:

1. The VTEC of GIMs is assessed during 15 years, i.e. for more than one solar cycle, vs +190 millions VTEC altimeter measurements, for TOPEX, JASON1 and JASON2:

- Such direct VTEC assessment has been performed in the worse case scenario (over the seas, typically very far from permanent GPS receivers).

- The GIMs of resumed and new IAACs, EMRG (from day 117, 2015), CASG, WHRG \& WHUG, show relative errors of 21-26\%. They are comparable with the ones of classical IGS IAACs, CODE, ESA, JPL 
and UPC, with relative errors of 20-26\%. The 15-min tomo-kriging UPC GIM (UQRG) has been excluded from these numbers since it is yet not available for the full period.

2. The STEC is assessed over more than 50 independent GNSS worldwide receivers, not used to compute any of the GIMs during two equinox and two solstice days in 2015. Indeed:

- Such direct STEC assessment is performed vs observed dSTEC values, including places not far from GNSS receivers used in the GIM computation.

- The STEC assessment indicates an overall agreement between different centers, with predominant errors around 20-30\%, with the exception of the Southern Hemisphere, South Pacific Ocean in particular, for some centers.

In short, this long-term study shows there is a general good agreement in the values between the different GIM techniques and implementations. Specially, it is worth to notice that the new IAACs contributing to IGS, produce GIMs inside the performance range of the classical IGS's IAACs and in some cases with better results than most of them. In this sense, this result opens the door to a potential improvement for IGSG, when passing from the combination of four consistent to seven consistent independent GIMs. The authors consider that increasing the number of contributing IAACs is very convenient for the following reasons:

1. Accuracy: Even when in general, as shown in this work, there is a very good agreement in the values from the GIMs, in some difficult scenarios (e.g. high ionospheric variability) there may be differences in the GIMs accuracy. In this sense, the combined GIM could apply the principle that in a large enough data set the majority will be more close to the real value. On the other hand, currently with only four GIMs it is very difficult to claim that we have a large enough data set to be statistically relevant. We think that seven is still a low number in this sense, but it is in any case a step forward in the right direction.

2. Reliability: At some point an IAAC can suffer from some downtime, in terms of connectivity or computing time. In this case, the degradation suffered by the IGS combined GIM will be lower if there are more contributors.

3. Community: It is also worth to remember that the IGS is not only about generating quality data, it is also about connecting experts working worldwide in GNSS. Furthermore, having more IAAC actively participating in IGS will help raising awareness of the its task and challenges.

Finally, we have shown that the increase of GIM time update (from 15 minutes to 1 and 2 hours), when a sun-fixed reference frame is used, produces a marginal degradation (just from $17.8 \%$ to $18.4 \%$ of relative error of worsening for UPC products). However the change of the technique, just one part, like the final interpolation in the case of UPC, produces a more significant improvement: from 19.8\%, with tomo-splines UPC final technique (UPCG, [16]) 
to $18.4 \%$, tomo-kriging, UPC rapid GIM UQRG. This one presents a better performance maintained in different comparisons ([31]) in the case of the 2hours time interval compared UPC GIMs. In summary, this states clearly that the interpolation technique used to generate the GIM has a much larger impact on the final result than the time update of the GIM when using a Sun-fixed reference frame. The reason is that in such reference frame the evolution of the ionosphere is typically slow. On the other hand, when using a Earth-fixed reference frame, the GIM time update has a much larger impact on the final resolution [35].

Last but not least, this work illustrates, similarly to [22], the discriminant capability of using strictly independent and relevant (direct) reference data in order to assess the global ionospheric maps:

- Either vertically (VTEC-altimeter) or in slant directions, most sensitive to mapping function suitability (dSTEC-GPS).

- Testing interpolation strategies, either typically far (with altimeter data over the seas) or typically close (with dSTEC-GPS) from the GNSS receivers used to compute the different GIMs.

\section{Acknowledgment}

This work has been possible thanks to the collaborative and friendly framework of the International GNSS Service, an organization providing first class open data \& open products to the scientific and technical GNSS communities (see [4]). We appreciate the editorial inputs of Dr. René Zandbergen from ESA/ESOC.

\section{References}

1. Abe, O., Villamide, X.O., Paparini, C., Radicella, S., Nava, B., Rodríguez-Bouza, M.: Performance evaluation of gnss-tec estimation techniques at the grid point in middle and low latitudes during different geomagnetic conditions. Journal of Geodesy 91(4), 409-417 (2017)

2. Azpilicueta, F., Brunini, C.: Analysis of the bias between TOPEX and GPS vTEC determinations. Journal of Geodesy 83(2), 121-127 (2009)

3. Dach, R., Schaer, S., Arnold, D., Orliac, E., Prange, L., Susnik, A., Villiger, A., Jäggi, A.: CODE final product series for the IGS. URL: http://www.aiub.unibe.ch/download/CODE p. DOI: 10.7892/boris.75876 (2016)

4. Dow, J.M., Neilan, R., Rizos, C.: The international GNSS service in a changing landscape of global navigation satellite systems. Journal of Geodesy 83(3-4), 191-198 (2009)

5. Feltens, J.: Development of a new three-dimensional mathematical ionosphere model at European Space Agency/European Space Operations Centre. Space Weather 5(12) (2007)

6. Feltens, J., Angling, M., Jackson-Booth, N., Jakowski, N., Hoque, M., HernándezPajares, M., Aragón-Àngel, A., Orús, R., Zandbergen, R.: Comparative testing of four ionospheric models driven with GPS measurements. Radio Science 46(6) (2011)

7. Georgiadou, P.: Modelling the ionosphere for an active control network of gps stations (1994) 
8. Gerzen, T., Feltens, J., Jakowski, N., Galkin, I., Denton, R., Reinisch, B., Zandbergen, R.: Validation of plasmasphere electron density reconstructions derived from data on board CHAMP by IMAGE/RPI data. Advances in Space Research 55(1), 170-183 (2015)

9. Gerzen, T., Feltens, J., Jakowski, N., Galkin, I., Reinisch, B., Zandbergen, R.: Analysis of the IMAGE RPI electron density data and CHAMP plasmasphere electron density reconstructions with focus on plasmasphere modelling. Advances in Space Research 58(6), 856-866 (2016)

10. Ghoddousi-Fard, R.: GPS ionospheric mapping at Natural Resources Canada. In: IGS Workshop, Pasadena (2014)

11. Ghoddousi-Fard, R., Héroux, P., Danskin, D., Boteler, D.: Developing a GPS TEC mapping service over Canada. Space Weather 9(6) (2011)

12. Ghoddousi-Fard, R., Prikryl, P., Lahaye, F.: GPS phase difference variation statistics: A comparison between phase scintillation index and proxy indices. Advances in Space Research 52(8), 1397-1405 (2013)

13. Hernández-Pajares, M.: IGS Ionosphere WG Status Report: Performance of IGS Ionosphere TEC Maps-Position Paper. In: IGS Workshop, Bern (2004)

14. Hernández-Pajares, M., Garcia-Fernández, M., Rius, A., Notarpietro, R., Engeln, A.v., Olivares-Pulido, G., Aragón-Ángel, Á., García-Rigo, A.: Electron density extrapolation above F2 peak by the linear Vary-Chap model supporting new GNSS-LEO occultation missions. Journal of Geophysical Research: Space Physics pp. n/a-n/a. DOI 10.1002/2017JA023876. URL http://dx.doi.org/10.1002/2017JA023876. 2017JA023876

15. Hernández-Pajares, M., Juan, J., Sanz, J.: Neural network modeling of the ionospheric electron content at global scale using GPS data. Radio Science 32(3), 1081-1089 (1997)

16. Hernández-Pajares, M., Juan, J., Sanz, J.: New approaches in global ionospheric determination using ground GPS data. Journal of Atmospheric and Solar-Terrestrial Physics 61(16), 1237-1247 (1999)

17. Hernández-Pajares, M., Juan, J., Sanz, J.: Improving the Abel inversion by adding ground GPS data to LEO radio occultations in ionospheric sounding. Geophysical Research Letters 27(16), 2473-2476 (2000)

18. Hernández-Pajares, M., Juan, J., Sanz, J., Colombo, O.L.: Application of ionospheric tomography to real-time GPS carrier-phase ambiguities Resolution, at scales of 400$1000 \mathrm{~km}$ and with high geomagnetic activity. Geophysical Research Letters 27(13), 2009-2012 (2000)

19. Hernández-Pajares, M., Juan, J., Sanz, J., Orus, R., García-Rigo, A., Feltens, J., Komjathy, A., Schaer, S., Krankowski, A.: The IGS VTEC maps: a reliable source of ionospheric information since 1998. Journal of Geodesy 83(3-4), 263-275 (2009)

20. Hernández-Pajares, M., Juan, J., Sanz, J., Sole, J.: Global observation of the ionospheric electronic response to solar events using ground and LEO GPS data. Journal of Geophysical Research: Space Physics 103(A9), 20,789-20,796 (1998)

21. Hernández-Pajares, M., Juan, J.M., Sanz, J., Aragón-Àngel, À., García-Rigo, A., Salazar, D., Escudero, M.: The ionosphere: effects, GPS modeling and the benefits for space geodetic techniques. Journal of Geodesy 85(12), 887-907 (2011)

22. Hernández-Pajares, M., Roma-Dollase, D., Krankowski, A., García-Rigo, A., OrúsPérez, R.: Methodology and consistency of slant and vertical assessments for ionospheric electron content models. Journal of Geodesy (doi:10.1007/s00190-017-1032-z), 1-10 (2017)

23. Juan, J.M., Rius, A., Hernández-Pajares, M., Sanz, J.: A two-layer model of the ionosphere using Global Positioning System data. Geophysical Research Letters 24(4), 393-396 (1997)

24. Komjathy, A., Sparks, L., Wilson, B.D., Mannucci, A.J.: Automated daily processing of more than 1000 ground-based GPS receivers for studying intense ionospheric storms. Radio Science 40(6) (2005)

25. Komjathy, A., Wilson, B., Runge, T., Boulat, B., Mannucci, T., Pi, X., Reyes, M., Sparks, L.: A new ionospheric model for wide area differential GPS: the multi-shell approach. In: Proceedings of the ION National Technical Meeting, San Diego, CA, January 2830. The Institute of Navigation (ION) (2002) 
26. Li, Z., Yuan, Y., Wang, N., Hernandez-Pajares, M., Huo, X.: SHPTS: towards a new method for generating precise global ionospheric TEC map based on spherical harmonic and generalized trigonometric series functions. Journal of Geodesy 89(4), 331$345(2015)$

27. Luo, X., Xu, H., Li, Z., Zhang, T., Gao, J., Shen, Z., Yang, C., Wu, Z.: Accuracy assessment of the global ionospheric model over the southern ocean based on dynamic observation. Journal of Atmospheric and Solar-Terrestrial Physics 154, 127-131 (2017)

28. Mannucci, A., Wilson, B., Yuan, D., Ho, C., Lindqwister, U., Runge, T.: A Global mapping technique for GPS-derived ionospheric total electron content measurements. Radio science 33(3), 565-582 (1998)

29. Mannucci, A.J., Iijima, B., Sparks, L., Pi, X., Wilson, B., Lindqwister, U.: Assessment of global TEC mapping using a three-dimensional electron density model. Journal of Atmospheric and Solar-Terrestrial Physics 61(16), 1227-1236 (1999)

30. Mannucci, A.J., Wilson, B.D., Edwards, C.D.: A new method for monitoring the Earth's ionospheric total electron content using the GPS global network. In: Proceedings of the 6th International Technical Meeting of the Satellite Division of The Institute of Navigation (ION GPS 1993), Salt Lake City, UT, September 1993, pp. 1323-1332. The Institute of Navigation (ION) (1993)

31. Orús, R., Hernández-Pajares, M., Juan, J., Sanz, J.: Improvement of global ionospheric VTEC maps by using kriging interpolation technique. Journal of Atmospheric and Solar-Terrestrial Physics 67(16), 1598-1609 (2005)

32. Rovira-Garcia, A., Juan, J., Sanz, J., González-Casado, G., Ibáñez, D.: Accuracy of ionospheric models used in gnss and sbas: methodology and analysis. Journal of geodesy 90(3), 229-240 (2016)

33. Schaer, S.: Mapping and predicting the earths ionosphere using the Global Positioning System. 1999. 205p. Ph.D. thesis, Ph. D. dissertation. University of Bern, Bern, Switzerland (1999)

34. Schaer, S., Beutler, G., Rothacher, M., Springer, T.A.: Daily global ionosphere maps based on GPS carrier phase data routinely produced by the CODE Analysis Center. In: Proceedings of the IGS AC Workshop, Silver Spring, MD, USA, pp. 181-192 (1996)

35. Schaer, S., Gurtner, W., Feltens, J.: IONEX: The ionosphere map exchange format version 1. In: Proceedings of the IGS AC workshop, Darmstadt, Germany, vol. 9 (ftp://igs.org/pub/data/format/ionex1.pdf) (1998)

36. Vergados, P., Komjathy, A., Runge, T.F., Butala, M.D., Mannucci, A.J.: Characterization of the impact of GLONASS observables on receiver bias estimation for ionospheric studies. Radio Science 51(7), 1010-1021 (2016)

37. Yuan, Y., Ou, J.: Differential areas for differential stations (dads): a new method of establishing grid ionospheric model. Chinese Science Bulletin 47(12), 1033-1036 (2002)

38. Yuan, Y., Ou, J.: A generalized trigonometric series function model for determining ionospheric delay. Progress in Natural Science 14(11), 1010-1014 (2004)

39. Zhang, H., Xu, P., Han, W., Ge, M., Shi, C.: Eliminating negative VTEC in global ionosphere maps using inequality-constrained least squares. Advances in Space Research 51(6), 988-1000 (2013) 